\title{
Modelagem da Distribuição Potencial de Mangifera indica L. sob Cenários Climáticos Futuros no Bioma Caatinga
}

\author{
Marilângela da Silva Sobrinho ${ }^{1,4}$, Arnóbio de Mendonça Barreto Cavalcante ${ }^{2,4}$ (D), \\ Aryberg de Souza Duarte ${ }^{2}$, Geziel dos Santos de Sousa ${ }^{3}$ \\ ${ }^{1}$ Superintendência Estadual do Meio Ambiente, Fortaleza, CE, Brasil. \\ ${ }^{2}$ Instituto Nacional de Pesquisas Espaciais, Unidade de Eusébio, CE, Brasil. \\ ${ }^{3}$ Secretaria Municipal da Saúde, Fortaleza, CE, Brasil. \\ ${ }^{4}$ Mestrado Profissional em Climatologia e Aplicações nos Países da CPLP e África, \\ Universidade Estadual do Ceará, Fortaleza, CE, Brasil.
}

Recebido em: 30 de Abril de 2019 - Aceito em: 17 de Maio de 2019

\begin{abstract}
Resumo
Mangifera indica L. (mangueira) é uma planta exótica invasora na região Nordeste do Brasil. No estado do Ceará sua presença merece particular atenção na Área de Proteção Ambiental (APA) da Serra de Baturité (setor norte do Estado). O sinergismo da bioinvasão com o aquecimento do sistema climático somado ao parco conhecimento sobre a distribuição geográfica local dessa espécie, limita qualquer iniciativa de controle de invasões no futuro. Os objetivos desse estudo foram: 1) modelar a distribuição geográfica potencial de áreas climaticamente adequadas para a $M$. indica no bioma Caatinga em cenários climáticos futuros e; 2) avaliar a dinâmica espaço-temporal das áreas projetadas, com destaque para a APA da Serra de Baturité. Para a modelagem utilizou-se do algoritmo MaxEnt, das fatias de tempo 1961-1990 (estado considerado como atual do clima), 2041-2060 (2050) e 2061-2080 (2070) e dos cenários RCP 4.5 e 8.5 do CMIP5. Cinco modelos foram gerados dos quais, os modelos para 2070 projetaram contração acima de $50 \%$ para as áreas de alto potencial de ocorrência da espécie-alvo.
\end{abstract}

Palavras-chave: mudanças climáticas, planta exótica, mangueira.

\section{Modeling the Potential Distribution of Mangifera indica L. under Future Climate Scenarios in the Caatinga Biome}

\begin{abstract}
Mangifera indica L. (mangueira) is an invasive exotic plant in the Northeast region of Brazil. In the state of Ceará, its presence deserves special attention in the Environmental Protection Area (EPA) of the Serra de Baturite (north sector of state). The synergism of bioinvasion with the warming of the climatic system added to the lack of knowledge about the local geographic distribution of this species limits any invasion control initiatives in the future. The objectives of this study were: 1) to model the potential geographical distribution of climatically adequate areas for $M$. indica in the Caatinga biome in future climatic scenarios; 2) to evaluate the spatiotemporal dynamics of the projected areas, with emphasis on the EPA of Serra de Baturité. For the modeling, we used the MaxEnt algorithm, the time slices 1961-1990 (actual state of climate), 2041-2060 (2050) and 2061-2080 (2070) and the RCP 4.5 and 8.5 scenarios of CMIP5. Five models were generated from which the models for 2070 projected contraction above $50 \%$ for areas of high potential occurrence of the target species.
\end{abstract}

Keywords: climate change, exotic plant, mangueira.

Autor de correspondência: Arnóbio de M. B. Cavalcante, arnobio.cavalcante@inpe.br. 


\section{Introdução}

Os seres humanos transportam espécies pelo mundo há séculos. Entre as razões para a introdução de espécies em regiões onde outrora não existiam estão a agricultura, silvicultura, forragem e horticultura (Zenni, 2013). Como consequência, hoje, praticamente todos os ecossistemas do mundo hospedam espécies exóticas (Van Kleunen et al., 2015).

As espécies exóticas quando passam a invadir ambientes naturais podem reduzir a biodiversidade local (Gilbert e Levine, 2013), alterar processos ecossistêmicos (Vilá et al., 2011; Katsanevakis et al., 2014) e trazer prejuízos socioeconômicos (Reaser et al., 2007). As espécies exóticas invasoras são uma das mais severas ameaças à conservação de espécies nativas, comunidades e ecossistemas (Vitousek et al., 1996; Wilcove et al., 1998; Traveset e Richardson, 2006), sendo esse fenômeno considerado a segunda maior causa de depauperização da biodiversidade no mundo (Leão et.al., 2011).

No Brasil, os primeiros registros oficiais de invasões biológicas datam de 1824, com a observação da expansão populacional gradativa do capim africano Melinis minutiflora P. Beauv (Ziller et al., 2007). No Brasil existem mais de 117 espécies de plantas exóticas reconhecidas como estabelecidas e com potencial invasor ou invasoras. Dentre elas consta a Mangifera indica L., doravante mangueira (Op. cit.).

A chegada da mangueira ao Brasil aconteceu, supostamente, acompanhando as grandes navegações iniciadas a partir do século XV lideradas pelos portugueses, que possibilitaram a abertura do comércio entre a Ásia e Europa. Durante as transações comerciais ocorridas à época, mudas de mangueira foram transportadas de Goa, na Índia, onde a espécie é nativa, para o Leste/Oeste da África e, em seguida, para o Brasil (Cunha et al., 2002). No Brasil, a mangueira primeiro desembarcou no estado da Bahia, sendo introduzida e distribuída para o interior pelos índios e bandeirantes (Favero, 2008).

Atualmente, a planta está presente em todas as regiões do país, concentrando-se nas regiões Sudeste e Nordeste (IBGE, 2015). O clima quente e úmido e os períodos secos melhor definidos tem favorecido o predomínio da mangueira nessa última região (Caribe e Campos, 1991), sendo a mangueira classificada no tipo espécie exótica invasora de médio risco de invasão (Leão et al., 2011).

No estado do Ceará a mangueira já ocorre em diversas paisagens, merecendo destaque a Área de Proteção Ambiental (APA) da Serra de Baturité (Ceará, 2016), uma área detentora de paisagem de exceção ou enclave úmido, que se destaca das depressões sertanejas ao seu redor, devido o seu clima ameno, fator relacionado à sua localização e altitude (Ab’Sáber, 2003; Cavalcante, 2005). Nessa unidade de conservação não só a mangueira está presente, como também outras espécies exóticas invasoras e não invasoras (Cavalcante et al., 2011). A APA da Serra de Baturite é uma área estratégica do ponto de vista biológico e hídrico para o Ceará. Vale destacar também que a mangueira é uma espécie exótica invasora em muitas outras Unidades de Conservação (UCs) do país (Base, 2018).

As UCs são importantes repositórios da biodiversidade nativa, além de oferecer proteção para o fornecimento contínuo de processos e serviços ecossistêmicos. Essas áreas são altamente valorizadas pela sua conservação e significado cultural. A gestão de espécies invasoras tem alta prioridade dentro das UCs, onde os prejuízos são ainda maiores reconhecidos os propósitos para as quais elas foram criadas. A integridade das UCs atuais e futuras devem ser administradas diante de uma série de ameaças, dentre elas as bioinvasões, as mudanças climáticas e o sinergismo potencial dessas ameaças, que muitas vezes, os efeitos negativos desses fatores sinérgicos é de grande severidade para sistemas naturais (Didham et al., 2007).

Tendo em vista que o tema mudanças climáticas associado ao aquecimento global ganhou atenção mundial devido, dentre outras razões, aos impactos negativos imediatos e futuros sobre a biodiversidade, revelado por publicações nos últimos anos (Bellard et al., 2012; IPCC, 2014; Pecl et al., 2017), tem-se buscado ferramentas para revelar caminhos de possíveis mudanças na faixa de distribuição de espécies exóticas invasoras, beneficiadas pelas mudanças climáticas. E, uma ferramenta que pode fornecer uma compreensão preliminar nessa abordagem são os modelos de distribuição geográfica de espécie.

Esses modelos associam as localizações de uma espécie para um conjunto de variáveis ambientais e são projetados para prever a distribuição da espécie. A premissa central dessa abordagem é que, o melhor indicador das exigências climáticas de uma espécie é a sua atual área de distribuição (Guisan e Zimmermann, 2000; Guisan e Thuiller, 2005) e essa espécie só será capaz de estabelecerse em outras áreas com as mesmas condições climáticas.

Os modelos de distribuição de espécie tem sido usados para investigar as distribuições de diferente espécies exóticas invasoras e, embora o impacto delas em áreas protegidas tenha sido documentado há muito tempo (Obermeijer, 1937; Richardson, 1998; Mckinney, 2002), a compreensão das relações entre elas e mudanças do clima ainda é incipiente. Admite-se que as mudanças climáticas podem aumentar a capacidade de muitas espécies exóticas invasoras de invadir novas áreas ao mesmo tempo que diminuem a resistência dos ecossistemas naturais à invasão (Thuiller et al., 2005).

Nesse Contexto, levando-se em consideração a ação conjunta da bioinvasão com o aquecimento do sistema climático, que podem potencializar impactos negativos sobre a biodiversidade, bem como o parco conhecimento local sobre o modus operandi da espécie-alvo, isso torna limitante qualquer iniciativa de controle de invasões no futuro. Um primeiro passo crítico para enfrentar essa situação é 
munir-se de mais conhecimento. Portanto, os objetivos desse estudo foram: 1) modelar a distribuição geográfica de áreas climaticamente adequadas para a presença da mangueira no bioma Caatinga em cenários climáticos futuros e; 2) avaliar a dinâmica espaço-temporal das áreas projetadas, com destaque para a APA da Serra de Baturité (CE). Espera-se contribuir para uma primeira aproximação da dinâmica espaço-temporal da mangueira no médio e longo prazos, fornecendo aos gestores ambientais, particularmente, da APA da Serra de Baturité (CE), informações para uma avaliação de risco.

\section{Material e Métodos}

\section{1. Área de estudo}

A área de projeção do modelo corresponde ao bioma Caatinga, que está situado na sua quase totalidade na região Nordeste do Brasil com uma área de $844.453 \mathrm{~km}^{2}$ ou $10 \%$ do território Nacional (MMA, 2018). Essencialmente, o bioma Caatinga está revestido por uma vegetação submetida a um clima semiárido, cuja flora apresenta adaptações à deficiência hídrica e significativo endemismo (Rodal e Sampaio, 2002). Ademais, abriga pequenos enclaves de outros tipos de vegetação, do tipo mata serrana (APA da Serra de Baturité), cerrados e campos rupestres (Giullieti et al., 2006).

Inserida numa área de particular interesse no bioma Caatinga, a serra de Baturité é um aglomerado de elevações, situado na porção Nordeste do estado do Ceará, com coordenadas geográficas extremas entre $4^{\circ} 02^{\prime}$ a $4^{\circ} 32^{\prime}$ de latitude sul e $38^{\circ} 41^{\prime}$ a $39^{\circ} 07^{\prime}$ de longitude oeste, ocupando 38.220 ha. Desse total, a partir da cota $600 \mathrm{~m}$, aproximados 32.690 ha estão revestidos de mata atlântica, correspondendo à Área de Proteção Ambiental (APA), criada em 18 de setembro de 1990, através do Decreto Estadual $\mathrm{N}^{\circ} 20.956$ (Cavalcante et al., 2011).

\subsection{Espécie selecionada}

A espécie-alvo (mangueira) é originária da Índia e arquipélago Malaio com base nos registros de tempos remotos das variedades cultivadas, no número de nomes antigos, nas evidências fósseis, classificações taxonômicas, modo de germinação, abundância e até nos antigos relatos de viajantes e registros históricos (Mukherjee e Litz, 2009). Sua distribuição atual concentra-se entre os trópicos de Câncer e Capricórnio, nas latitudes de $20^{\circ} \mathrm{N} \mathrm{a}$ $20^{\circ} \mathrm{S}$, estando presente em quase 100 países (Mukherjee, 1985 apudLima Filho et al., 2002). A distribuição atual da mangueira evidencia que a espécie é altamente adaptável a diferentes ambientes. Além de ser abundante em sua área nativa, atua como espécie exótica invasora em várias partes no mundo, assim, estando presente na Ásia, África, América do Sul e do Norte e Oceania (Sandoval e Rodríguez, 2014).
No Brasil a mangueira é considerada exótica invasora, bem como na região Nordeste (Leão et al., 2011). Nessa região a espécie-alvo está presente em todos os estados e, no Ceará ocorre em alguns municípios e em todos os que compõem a APA da Serra de Baturité, significando dizer que a mangueira está presente em toda a APA (IBGE, 2015).

Conforme Instituto Hórus (Base, 2018) no bioma Caatinga a mangueira invade preferencialmente áreas ciliares. Sua invasão nesses ambientes compromete os espaços para ocupação de espécies nativas, além de provocar alteração no $\mathrm{pH}$ da água, devido ao apodrecimento de folhas e frutos que são produzidos em abundância. Também, leva impacto sobre a dispersão de espécies nativas zoocóricas, uma vez que é muito utilizada como fonte de alimento por animais, diminuindo o consumo de frutos de outras espécies e, consequentemente, a dispersão das espécies nativas.

\subsection{Registros de ocorrência global, regional e na APA}

Os registros de ocorrência da espécie-alvo global e regional na forma de coordenadas geográficas decimais foram obtidos a partir de três bancos de dados online, o Global Biodiversity Information Facility, SpeciesLink e o Instituto Hórus. Utilizou-se de 91 pontos de presença do Sudeste asiático e 31 pontos no bioma Caatinga. Para os registros de presença da mangueira em campo, estes foram tomados aleatoriamente na APA da Serra de Baturité, por meio de receptor GPS no período de 17 a 19 de março de 2018. Foram percorridas as principais rodovias que cortam a APA, incluindo as trilhas no interior da mata, totalizando $188 \mathrm{~km}$ amostrados. Além dos registros de ocorrência, foram realizadas observações das particularidades do ambiente.

\subsection{Preditores ambientais}

Quanto ao conjunto das variáveis ambientais para uso na modelagem, utilizou-se um total de oito variáveis climáticas, uma topográfica e o solo. Para as variáveis climáticas (Bio4 - coeficiente de variação da temperatura, Bio5 - temperatura máxima do mês mais quente, Bio9 temperatura média do trimestre mais seco, Bio12 - precipitação anual, Bio13 - precipitação do mês mais úmido, Bio16 - precipitação do trimestre mais úmido, Bio18 precipitação do trimestre mais quente, Bio19 - precipitação do trimestre mais frio), os dados foram baixados a partir de Worldclim (v. 1.4), com resolução espacial de 30 segundos $(\sim 1 \mathrm{~km})$. Para a variável topográfica (altitude) e de solo os dados foram obtidos no INPE/AMBDATA. Para a seleção dessas variáveis levou-se em conta a efetividade da variável ambiental em disparar e encerrar eventos fisiológicos, bem como em dispor espacialmente as plantas no bioma Caatinga. Isso foi levado a cabo por meio da literatura especializada (Ab' Sáber, 2003; 
Cavalcante, 2005; Sampaio, 2010; Albuquerque et al., 2012; Santos et al., 2014).

Uma Análise de Componentes Principais (Principal Component Analysis - PCA) foi aplicada, considerando o nível máximo de correlação entre as variáveis ambientais de 0,7 (Warren et al., 2014). Complementando, utilizou-se do teste Jackknife para calcular a importância relativa das variáveis supracitadas para os modelos de distribuição da espécie-alvo.

Para as condições climáticas futuras se utilizou de dados do modelo HadGEM2-ES, considerando as fatias de tempo 2041-2060 e 2061-2080 centradas, respectivamente, em 2050 e 2070 (Hijmans et al., 2005) e os cenários RCP 4.5 e 8.5 (Van Vuuren et al., 2011). Para as condições climáticas consideradas como clima atual foram tomados os dados observados para o período de 19601990 (Hijmans et al., 2005).

\subsection{Modelagem}

Os programas utilizados na modelagem foram o MaxEnt (v. 3.4.1) e o QGIS (v. 2.18). O teste estatístico da área sob a curva ROC (area under the curve - AUC) foi utilizado para validação ou avaliação da qualidade dos modelos finais gerados (Phillips et.al., 2006; Beaumont et al., 2009) levando-se em conta a classificação: AUC $\leq 0.80$ é um modelo pobre ou nulo; $0.81 \leq$ AUC $\leq$ 0.90 é adequado; $0.90<\mathrm{AUC} \leq 0.95$ é bom e $0.95<$ AUC $\leq 1.00$ é um excelente modelo (Thuiller et al., 2006). Ademais, foi calculada a extensão de áreas originalmente ocupadas, estendidas e contraídas da espéciealvo usando um SIG, desde o presente e à medida que o clima mudasse.

\subsection{Medidas de controle}

Realizou-se uma busca e seleção por medidas exitosas de controle de invasão para a mangueira no mundo, tendo em vista oferecer aos gestores do bioma Caatinga, bem como da APA da Serra de Baturité, recomendações para enfrentar possíveis ameaças de invasão da mangueira impulsionada pelas mudanças climáticas.

\section{Resultados e Discussão}

Considerando o conjunto das 10 variáveis ambientais mais independentes e com maior poder de contribuição para a distribuição da espécie-alvo, o teste Jackknife indicou que o solo (Sol), precipitação do trimestre mais frio (Bio19), temperatura máxima do mês mais quente (Bio5) e o coeficiente de variação da temperatura (Bio4) foram as variáveis ambientais de maior importância relativa para a modelagem da distribuição de $M$. indica. As contribuições individualizadas das variáveis supracitadas variaram de $9-30 \%$ e quando tomadas conjuntamente, superaram $65 \%$ em todos os modelos gerados. Por sua vez, as variáveis que menos contribuíram foram, no geral, a precipitação do trimestre mais úmido (Bio16) e precipitação do mês mais úmido (Bio13), com contribuições em torno de $4 \%$. As demais variáveis ambientais: altitude (Alt), temperatura média do trimestre mais seco (Bio9) e precipitação anual (Bio12) tiveram suas contribuições variando entre $4-10 \%$, excetuando-se a variável precipitação do trimestre mais quente (Bio18), cuja contribuição variou de $3-7 \%$.

Um total de cinco modelos finais foram produzidos para a distribuição de $M$. indica: um modelo de distribuição corrente (Figs. 1a e 2a - imagem duplicada), dois modelos de distribuição para 2050 (Figs. 1b e 2b) e dois

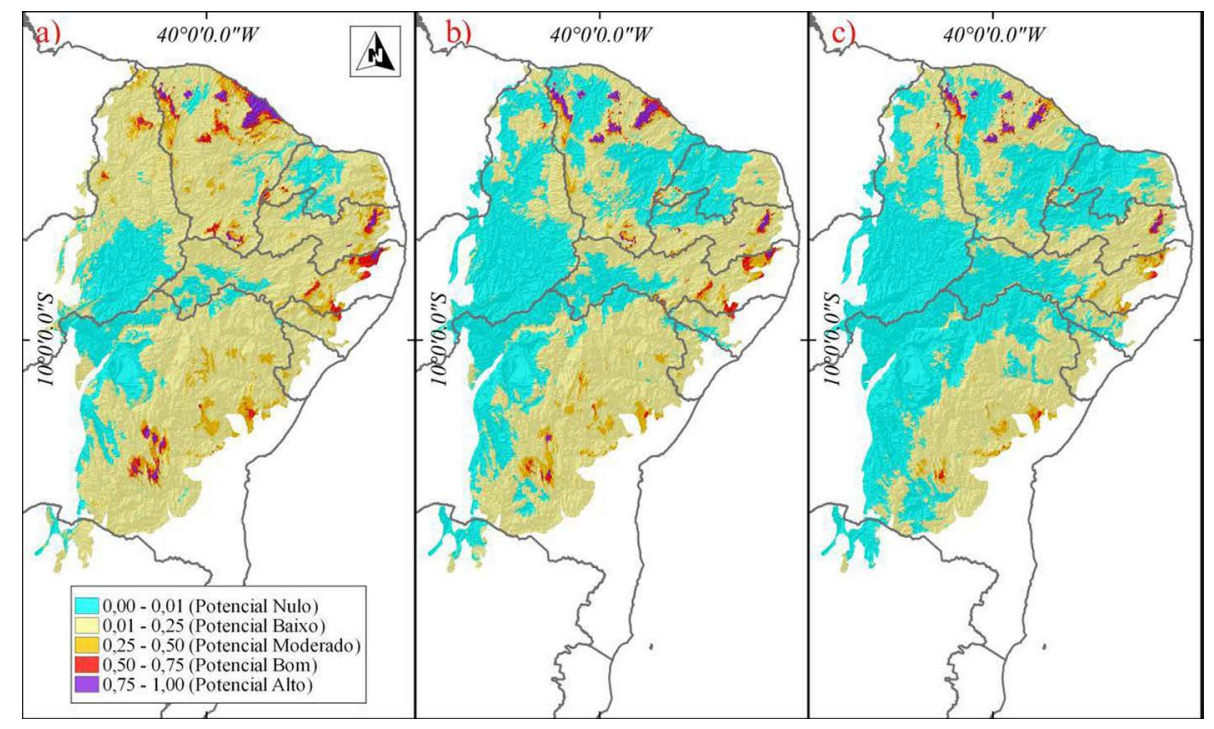

Figura 1 - Distribuição do espaço corrente (a) e dos espaços projetados para 2050 (b) e 2070 (c) sob o cenário RCP 4.5 para Mangifera indica no bioma Caatinga. 


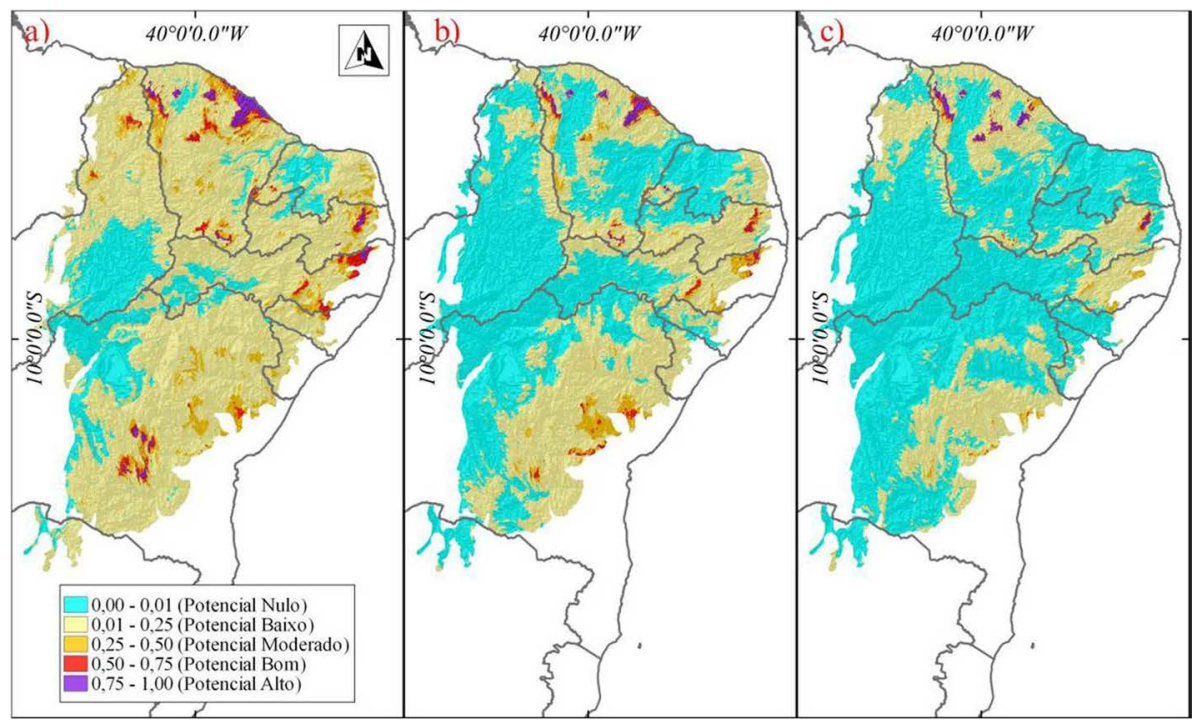

Figura 2 - Distribuição do espaço corrente (a) e dos espaços projetados para 2050 (b) e 2070 (c) sob o cenário RCP 8.5 para Mangifera indica no bioma Caatinga.

modelos de distribuição para 2070 (Figs. 1c e 2c). Os modelos finais tiveram precisão "bom" com valores entre $0.90 \leq \mathrm{AUC} \leq 0.95$.

O modelo de distribuição corrente revelou que existe uma gradação de probabilidade de ocorrência para a $M$. indica de zero (ausência) a 1,00 (presença) no bioma Caatinga (Figs. 1a e 2a). Também, delineou áreas de diferentes potenciais, sugerindo outras áreas geográficas, além daquelas atualmente com registros de presença. Destacando somente as áreas de alto potencial de ocorrência (>0.75 de chance de presença), estas somam $9.205,3 \mathrm{~km}^{2}$ (Tabela 1) correspondendo a $1,1 \%$ da área total do bioma $\left(844.453 \mathrm{~km}^{2}\right)$. O estado do Ceará concentrou a maior parte dessas áreas (Figs. 1a e 2a - imagem duplicada).

Para o futuro, os quatro modelos de distribuição produzidos (Figs. 1bc e 2bc) mostraram diferenças na distribuição potencial da $M$. indica, quando comparados entre si e ao modelo de distribuição corrente (Figs. 1a e 2a). Apesar das diferenças nos arranjos biogeográficos, os modelos de distribuição RCP 4.5 (2050 e 2070) e RCP 8.5 (2050 e 2070) foram semelhantes ao apontarem para uma contração das áreas mais adequadas para espécie-alvo, com o avançar dos anos até o final desse século (Tabela 1).
A modelagem bioclimática com as modificações climáticas inclusas permitiu identificar três cenários futuros distintos para a distribuição da $M$. indica em relação às condições atuais. No primeiro cenário, supostamente, um cenário de menor impacto no aumento da temperatura e na redução da precipitação, correspondendo aproximadamente ao ano RCP4.5/2050 (Fig. 1b), observou-se uma significativa contração das áreas de potencial alto $(>0,75)$ em cerca de $2 \mathrm{mil} \mathrm{km}^{2}$, isto é, envolvendo perdas de $19 \%$ da área original (Tabela 1). Em relação ao segundo cenário identificado, intermediário e correspondendo ao ano RCP8.5/2050 (Fig. 2b), já se verificou uma forte contração de $44 \%$ em perdas de áreas de potencial alto (Tabela 1). No terceiro e último cenário, correspondendo aproximadamente ao ano RCP4.5/2070 (Fig. 1c) e RCP8.5/2070 (Fig. 2c), observou-se uma drástica contração das áreas de maiores chances de ocorrência para a espécie, com perdas superando $50 \%$. Portanto, para os três cenários futuros e referenciados nas condições atuais, as áreas de potencial alto projetadas para a presença da $M$. indica no bioma Caatinga declinaram, podendo estas áreas no final desse século, restringir-se a uma área de aproximadamente 4 mil $\mathrm{km}^{2}$, situadas na sua quase totalidade no Norte do bioma.

Tabela 1 - Valores absolutos e relativos para as áreas de alto potencial de presença (>0,75 de chance de presença) da espécie-alvo em relação ao estado do clima no bioma Caatinga.

\begin{tabular}{|c|c|c|c|c|c|c|}
\hline \multirow[t]{3}{*}{ Espécie } & \multirow[b]{3}{*}{ Intervalo (ano) } & \multicolumn{5}{|c|}{ Condição climática } \\
\hline & & \multirow{2}{*}{$\frac{\text { Corrente }}{1961-1990(-)}$} & \multicolumn{2}{|c|}{$\mathrm{RCP} 4.5$} & \multicolumn{2}{|c|}{$\mathrm{RCP} 8.5$} \\
\hline & & & $2041-2060(2050)$ & $2061-2080(2070)$ & $2041-2060(2050)$ & $2061-2080(2070)$ \\
\hline \multirow[t]{2}{*}{ M. indica } & Área $\left(\mathrm{km}^{2}\right)$ & $9.205,3$ & $7.472,9$ & $4.344,7$ & $5.110,8$ & $4.400,7$ \\
\hline & Contração (\%) & - & 18,8 & 52,8 & 44,5 & 52,2 \\
\hline
\end{tabular}


Esse efeito contração revelado pelas simulações para $M$. indica, pode ser visto como um alerta de perigo ao desaparecimento local de uma planta importante ou, como uma oportunidade de controle de uma potencial bioinvasora. Alerta, porque sinaliza para o declínio de áreas potenciais para a ocorrência e cultivo da planta que, consequentemente, terá impacto sócio-econômico. Ademais, porque permite inferir para o desaparecimento de outras espécies vegetais com exigências ambientais semelhantes a da M. indica. Por sua vez, é uma oportunidade porque facilita o controle da bioinvasora em áreas onde sua presença é indesejada, por exemplo nas UCs. Essa última perspectiva é melhor observada na porção meridional do bioma, aproximadamente na fronteira do estado de Pernambuco com a Bahia para baixo, cuja contração da área de potencial alto desapareceu totalmente em 2070.

Com relação às áreas remanescentes predominantes de alto potencial e levando-se em conta a dinâmica espaço-temporal delas no bioma (Figs. 1 e 2), estas tenderão a concentrar-se no Norte do estado do Ceará (porção mais setentrional do bioma) e em áreas elevadas como a APA da Serra de Baturité. Essas elevações por estarem localizadas próximas do oceano Atlântico, de onde partem fortes ventos carregados de umidade que as atingem durante todo o ano, tem seus aspectos meteorológicos (temperatura e precipitação) diferenciados, com impactos na cobertura vegetal e segurança hídrica requerida pela espécie-alvo. Essa conjugação do relevo com a temperatura e o regime de chuvas favorece a presença, não só da espéciealvo que tem porte arbóreo, como também de formações florestais. Essas formações florestais são áreas de florestas tropicais úmidas encravadas no bioma Caatinga, conhecidas como paisagens de exceção (Ab'Sáber, 2003), brejos nordestinos ou matas serranas (Cavalcante, 2005).

Na APA da Serra de Baturité (32.690 ha) o número de indivíduos de $M$. indica amostrados alcançou 1.291 indivíduos, ocorrendo desde áreas adjacentes às rodovias, áreas degradadas e matas secundárias, até no interior de matas mais preservadas. Este número desperta preocupação, pois significa forte presença da bioinvasora na APA. São cerca de $25 \mathrm{ind} / \mathrm{ha}$ e, considerando os $188 \mathrm{~km}$ percorridos, ter-se-ia uma média de sete (7) indivíduos da espécie-alvo em média por quilômetro linear.

Portanto, em confirmando-se as mudanças climáticas preditas, considerando a possibilidade de impacto negativo da bioinvasora impulsionada pelas mudanças climáticas e observando os modelos de distribuição futuros produzidos, que apontaram para uma concentração das áreas de potencial alto para $M$. indica no bioma Caatinga no Norte do estado do Ceará, particularmente, em elevações como a APA da Serra de Baturité, é razoável admitir a necessidade de medidas preventivas para controle da espécie-alvo para os anos vindouros, considerando uma perspectiva conservacionista da flora nativa local.
Diante do exposto e fazendo um esforço de síntese recomenda-se, de modo geral, as seguintes medidas preventivas para aplacar possíveis invasões da $M$. indica impulsionada por alterações climáticas no bioma Caatinga e, particularmente, na APA da Serra de Baturité: 1) impedir o desembarque de indivíduos da espécie-alvo em áreas onde ainda não foi observada sua presença; 2) instalar um código de conduta preventiva envolvendo todos os municípios da APA (rede de observação); 3) evitar ou reduzir o desenvolvimento de sementes de mangueiras em trilhas por meio da educação ambiental nas escolas e, 4) reflorestar com espécies nativas áreas desejáveis pela espécie invasora.

\section{Conclusões}

À medida que as mudanças climáticas avançam, o espaço climaticamente adequado e disponível para mangueira no bioma Caatinga é projetado para diminuir, embora essas áreas adequadas ainda permaneçam com relativa densidade espacial ao norte do bioma até pelo menos 2070. Apesar de grande parte dessas áreas remanescentes estarem inseridas em UCs, incluindo a APA da serra de Baturité, o risco de invasão pela mangueira continua alto, devido essas áreas serem as únicas adequadas para a presença da planta ou seu cultivo natural, sem irrigação. O número significativo de mangueiras encontradas na APA da Serra de Baturité, evidencia a suscetibilidade das áreas à invasão e, somando-se a isso, as condições ambientais ideais para o desenvolvimento da planta e o potencial de dispersão por humanos e animais.

A erradicação da espécie é inviável, uma vez que ela tem importância sócio-econômica e, também, é dispersa por animais silvestres. Como foi constatado pela modelagem que áreas continuarão a ser adequadas em cenários climáticos futuros até pelo menos 2070, reforça a necessidade de implementação de medidas adaptativas ou de manejo, junto a um programa de controle para minimizar a probabilidade da mangueira alcançar todo seu potencial invasivo.

Essas medidas tem a finalidade de reduzir a vulnerabilidade e potencializar a conservação da biodiversidade nativa, pois para um dado ecossistema, comunidades funcionalmente diversas são mais prováveis de se adaptarem à mudança e à variabilidade de clima do que comunidades empobrecidas (PNA, 2016).

Por fim, todos os biomas brasileiros sofrerão diferentes impactos em consequência das mudanças no clima e, embora o bioma Caatinga esteja dentre os menos afetados, dado sua dominância por vegetações abertas (PNA, 2016), perdas de biodiversidade poderão ocorrer, em parte, provocada por espécies exóticas invasoras beneficiadas por alterações no clima. Portanto, continuar modelando plantas potencialmente invasoras nessa perspectiva e levando-se em conta um bioma vulnerável, é importante 
termos predições de impactos mesmo que em primeira aproximação, que poderão auxiliar no direcionamento de pesquisas e de medidas preventivas de adaptação mais eficazes.

\section{Referências}

AB' SÁBER, A.N. Os Domínios de Natureza no Brasil: Potencialidades Paisagísticas. São Paulo: Ateliê Editorial, 2003. 160p.

ALBUQUERQUE, U.P.; ARAÚJO, E.L.; EL-DEIR, A.C.A.; LIMA, A.L.A.; SOUTO, A. et al. Caatinga revisited: ecology and conservation of an important seasonal dry forest. The Scientific World Journal, v. 2012, p. 1-18, 2012. Doi: 10.1100/2012/205182.

BASE DE DADOS NACIONAL DE ESPÉCIES EXÓTICAS INVASORAS I3N BRASIL, INSTITUTO HÓRUS DE DESENVOLVIMENTO E CONSERVAÇÃO AMBIENTAL, Florianópolis-SC. Disponível em: http://i3n.instituto horus.org.br/www. Acesso em 05 jun.2018.

BEAUMONT, L.J.; GALLAGHER, R.V.; DOWNEY, P.O.; THUILLER, W.; LEISHMAN, M.R.; HUGHES, L. Modelling the impact of Hieracium spp. on protected areas in Australia under future climates. Ecography, v. 32, p. 757764, 2009.

BELLARD, C.; BERTELSMEIER, C.; THUILLER, W.; COURCHAMP, F. Impacts of climate change on the future of biodiversity. Ecology Letters, v. 15, n. 4, p. 365- 377, 2012.

CARIBÉ, J.; CAMPOS, J.M. Plantas que Ajudam o Homem: Guia Prático para a Época Atual. São Paulo: Cultrix/ Pensamento, 1991.275p.

CAVALCANTE, A.M.B. Jardins Suspensos no Sertão. Scientific American Brasil, São Paulo, v. 32, p. 66-73, 2005.

CAVALCANTE, A.M.B.; OLIVEIRA, E.M.; SILVA SOBRINHO, M. Plantas Exóticas e Invasoras: Risco Potencial para a Gestão Ambiental da APA da Serra de Baturité. In: FREDERICO, H.B. (Org.). Serra de Baturité - Uma Visão Integrada das Questões Ambientais. Fortaleza: Expressão Gráfica e Editora, 2011. p 101-114.

CEARÁ (Estado). Lei: 16.002, 02 de maio de 2016. Cria o Programa de Valorização das Espécies Vegetais Nativas. Diário Oficial do Estado. Série 3, ano VIII, Nº81, pág. 10. 2016.

CUNHA, G.A.P.; PINTO, A.C.Q.; FERREIRA, F.R.. Origem, Dispersão, Taxonomia e Botânica. In: GENÚ, P.J.C.; PINTO, A.C.Q. (Org.). A Cultura da Mangueira. Brasília: Embrapa Informação Tecnológica, 2002. p. 32-36.

DIDHAM, R.K.; TYLIANAKIS, J.M.; GEMMELL, N.J.; RAND, T.A.; EWERS, R.M. Interactive effects of habitat modification and species invasion on native species decline. Science Direct, v. 22, p. 489-496, 2007.

FAVERO, L.A. A Cultura da Manga no São Francisco: Posicionamento, Limites, Oportunidades e Ações Estratégicas. Fortaleza: Banco do Nordeste do Brasil, 2008. 230p.

Gilbert, B.; Levine, J.M. Plant invasions and extinction debts. PNAS, v. 110, n. 5, p. 1744-1749, 2013.

GUILLIETI, A.M.; CONCEIÇÃO, A.; QUEIROZ, L.P. Diversidade e Caracterização das Fanerógamas do Semiárido
Brasileiro. Recife: Associação Plantas do Nordeste, 2006. 488p.

GUISAN, A.; THUILLER, W. Predicting species distribution: offering more than simple habitat models. Ecology Letters, v. 8, p. 993-1009, 2005.

GUISAN, A.; ZIMMERMANN, N. Predictive habitat distribution models in ecology. Science Direct, v. 135, p. 147-186, 2000.

HIJMANS, R.J.; CAMERON, S.E.; PARRA, J.L.; JONES, P.G.; JARVIS, A. Very high resolution interpolated climate surfaces for global land areas. International Journal of Climatology, v. 25, p. 1965-1978, 2005.

IBGE - Instituto Brasileiro de Geografia e Estatística. Produção Agrícola Municipal, 2015. Disponível em: http://www. ibge.gov.br/estatisticas-novoportal/economicas/agriculturae-pecuaria. Acesso em: 20 dez. 2017.

IPCC - Intergovernmental Panel on Climate Change. Climate Change 2014: Synthesis Report. Contribution of Working Groups I, II and III to the Fifth Assessment Report of the Intergovernmental Panel on Climate Change [Core Writing Team, R.K. Pachauri and L.A. Meyer (eds.)]. Geneva: IPCC, 2014. 151p.

KATSANEVAKIS, S.; COLL, M.; PIRODDI, C.; STEENBEEK, J.; BEM, F.; LASRAM, R. et al. Invading the Mediterranean Sea: biodiversity patterns shaped by human activities. Frontiers in Marine Science, v. 1, n. 32, p. 1-11, 2014.

LEÃO, T.C.C; ALMEIDA, W.R.; DECHOUM, M.; ZILLER, S. R. Espécies Exóticas Invasoras no Nordeste do Brasil: Contextualização, Manejo e Políticas Públicas. Recife: Cepan, 2011.99 p.

LIMA FILHO, J.M.P.; ASSIS, J.S.; TEIXEIRA, A.H.C.; CUNHA, G.A.P.; CASTRO NETO, M.T. Ecofisiologia. In: GENÚ, P.J.C.; PINTO, A.C.Q. (Org.). A Cultura da Mangueira. Brasília: Embrapa Informações Tecnológica, 2002. p. 38-49.

MCKINNEY, M.L. Influence of settlement time, human population, park shape and age, visitation and roads on the number of alien plant species in protected areas in the USA. Diversity and Distributions, v. 8, p. 311-318, 2002.

MINISTÉRIO DO MEIO AMBIENTE. Bioma Caatinga. Disponível em: http://www.mma.gov.br/biomas/caatinga. Acesso em: 29 mai.2018.

MUKHERJEE, S.K; LITZ, R.E. Introdution: Botany and Importance. In: LITZ, R.E. (Org.). The Mango: Botany, Production and Uses. Florida: Tropical Research and Education Center, 2009. p. 1-18.

OBERMEIJER, A.A. A preliminary list of the plants found in the Kruger National Park. Annals of the Transvaal Museum, v. 17, n. 4, p. 185 - 227, 1937.

PECL, G.T.; ARAUJO, M.B.; BELL, J.; BLANCHARD, J.; BONEBRAKE, T.C. et al. Biodiversity redistribution under climate change: Impacts on ecosystems and human well-being. Science, v. 355, n. 6332, p. 1-9, 2017.

PHILLIPS, S.J.; ANDERSON, R.P.; SCHAPIRE, R.E. Maximum entropy modeling of species geographic distributions. Ecological Modelling, v. 190, p. 231-259, 2006.

PNA - Plano Nacional de Adaptação à Mudança Climática. Volume 2: Estratégias Setoriais e Temáticas. Brasília: MMA, 2016. 295 p. 
REASER, J.K.; MEYERSON, L.A.; CRONK, Q.; POORTER, M.; ELDREGE, L.G. et al. Ecological and socioeconomic impacts of invasive alien species in island ecosystems. Environmental Conservation, v. 34, p. 88-111, 2007.

Richardson, D.M. Forestry Trees as Invasive Aliens. Conservation Biology, v. 12, n. 1, p. 18-26, 1998.

RODAL, M.J.N.; SAMPAIO, E.V.S.B. A vegetação do bioma Caatinga. In: SAMPAIO, E.V.S.B.; GIULIETTI, A.M.; VIRGÍNIO, J.; GAMARRA-ROJAS, C.F.L. (Org.). Vegetação e Flora da Caatinga. Recife; PNE/CNIP, 2002. p.11-14.

SAMPAIO, E.V.S.B. Caracterização do bioma Caatinga: características e potencialidades. In: GARIGLIO, M.A.; SAMPAIO, E.V.S.B.; CESTARO, L.A.; KAGEYAMA, P.Y. (Org.). Uso Sustentável e Conservação dos Recursos Florestais da Caatinga. Brasília: SFB, 2010. p.29-48.

SANDOVAL, J.R.; RODRÍGUEZ, P.A. Invasive Species Compedium. Wallingford: CABI, 2014.

SANTOS, M.G.; OLIVEIRA, M.T.; FIGUEIREDO, K, V.; FALÇÃO, H.M.; ARRUDA, E.C.P. et al. Caatinga, the Brazilian dry tropical forest: can it tolerate climate change? Theoretical and Experimental Plant Physiology, v. 26, p. 83-99, 2014. Doi: 10.1007/s40626-014-0008-0.

THUILLER, W.; BROENNIMANN, O.; HUGES, G.; ALKEMADE, J.R.M.; MIDGLEY, G.F.; CORSI, F. Vulnerability of African mammals to anthropogenic climate change under conservative land transformation assumptions. Global Change Biology, v. 12, n. 3, p. 424-440, 2006.

THUILLER W.; RICHARDSON D.M; KPYSEK P.; MIDGLEY G.F.; HUGHES, G.O.; ROUGET M. Niche-based modeling as a tool for predicting the risk of alien plant invasions at a global scale. Global Change Biology, v. 11, n. 12, p. 2234-2250, 2005.

TRAVESET, A.; RICHARDSON, D.M. Biological invasions as disruptors of plant reproductive mutualisms. Trends Ecology \& Evolution, v. 21, p. 208-216, 2006.

VAN KLEUNEN, M.; DAWSON, W. P.; PERGL, J.; ESSL, F. Global exchange and accumulation of non-native plants. Nature, v. 525, n. 7567, p. 100-103, 2015.

VAN VUREN, D.P.; EDMONDS. J.; KAINUMA, M.; RIAHI, K.; THOMSON, A. et al. The representative concentration pathways: an overview. Climatic Change, v. 109, p. 5-31, 2011.

VILÀ, M.; ESPINAR, J.L.; HEDJA, M.; HULME, P.E.; Jarosik, V. et al. Ecological impacts of invasive alien plants: a metaanalysis of their effects on species, communities and ecosystems. Ecology Letters, v. 14, n. 7, p. 702-708, 2011.

VITOUSEK, P.M.; D'ANTONIO, C.M.; LOOPE, L.L.; WESTBOOK, R. Biological invasions as global environmental change, American Scientist, v. 84, n. 5, p. 468-478, 1996.

WARREN, D.L.; WRIGHT, A.N.; SEIFERT, S.N.; SHAFFER, H.B. Incorporating model complexity and spatial sampling bias into ecological niche models of climate change risks faced by 90 California vertebrate species of concern. Biodiversity Research, v. 20, n. 3, p. 334-343, 2014.

WILCOVE, D.S; Rothstein, D.; Dubow, J.; Phillips A.; Losos, E. Quantifying Threats to Imperiled Species in the United States. BioSciense, v. 48, n. 8, p. 607-615, 1998.

ZENNI, R.D. Analysis of introduction history of invasive plants in Brazil reveals patterns of association between biogeographical origin and reason for introduction. Austral Ecology, v. 39, p. 401-407, 2013.

ZILLER, S.R.; ZALBA, S.M.; ZENNI, R.D. Modelo para o Desenvolvimento de uma Estratégia Nacional para Espécies Exóticas Invasoras. Programa de Espécies Invasoras para a América do Sul. Brasília: The Nature Conservancy, 2007. 57 p.

\section{Endereços de Internet}

Ambdata: www.dpi.inpe.br/Ambdata/mapa_solos.php Biodiversity Information Facility: www.gbif.org

Instituto Hórus: www.institutohorus.org.br

MaxEnt: http://biodiversityinformatics.amnh.org/open_source/ maxent

QGIS: www.qgis.org

SpeciesLink: www.splink.cria.org.br

Worldclim: worldclim.org

License information: This is an open-access article distributed under the terms of the Creative Commons Attribution License (type CC-BY), which permits unrestricted use, distribution and reproduction in any medium, provided the original article is properly cited. 